\title{
Modelo de desarrollo de las microempresas del grupo "EI Salinerito" en el Ecuador
}

\section{Model of development of the micro-enterprises of the "EI Salinerito" group in Ecuador}

Víctor H. Vizueta T.

Universidad Internacional del Ecuador, Ecuador

Ing. Joffre A. Santamaría Y. MBA

Universidad de Guayaquil, Ecuador

Ing. Vladimir A. Guerrero C. MBA

Universidad de Guayaquil, Ecuador

Isabel C. Mero V.

Universidad de Guayaquil, Ecuador

Autor por correspondencia: vivizuetato@uide.edu.ec, joffre.santamariay@ug.edu.ec, vladimir.guerrerov@ug.edu.ec, isabel.merovi@ug.edu.ec

Fecha de recepción: 26 de febrero de 2018 - Fecha de aceptación: 15 de agosto de 2018

Resumen: Mediante este artículo se procurará analizar el modelo de desarrollo de las microempresas del Grupo "El Salinerito" de la parroquia Salinas de Guaranda provincia de Bolívar en el Ecuador. La investigación realizada presenta algunos elementos que explican el modelo de desarrollo microempresarial como: el trabajo colectivo y solidario de los pobladores de la parroquia Salinas, la planificación de la producción agroindustrial basada en valores como el trabajo, cooperación, ahorro, autogestión, reinversión de sus utilidades y el emprendimiento social y la adecuada aplicación de los canales de distribución y comercialización de sus productos. El proceso de emprendimiento social de la parroquia Salinas y sus microempresas está orientada a los principios del Buen Vivir y a la reinversión de sus ganancias en obras sociales con la finalidad de mejorar el nivel de vida de sus pobladores.

Palabras Claves: modelo de desarrollo; microempresa; emprendimiento; cooperación

\begin{abstract}
Through this article, an attempt will be made to analyze the development model of the microenterprises of the "El Salinerito" Group of the Salinas de Guaranda parish in the province of Bolivar in Ecuador. The research carried out presents some elements that explain the model of microenterprise development as: the collective and solidary work of the residents of the Salinas parish, the planning of the agro-industrial production based on values such as work, cooperation, saving, self-management, reinvestment of their utilities and social entrepreneurship and the proper application of distribution channels and marketing of their products. The process of social entrepreneurship of the Salinas parish and its micro-enterprises is oriented towards the principles of Good Living and the reinvestment of its profits in social projects with the aim of improving the standard of living of its inhabitants.
\end{abstract}

Key words: development model; microenterprise; entrepreneurship; cooperation 


\section{Introducción}

La parroquia Salinas está ubicada en el cantón Guaranda de la Provincia de Bolívar en la república del Ecuador, debe su nombre a las minas de sal que desde tiempos inmemoriales eran explotadas por sus habitantes antes de la colonia española. Desde 1884 en que fue creada como parroquia civil, sus habitantes en su mayoría indígenas y mestizos, vivían de la extracción, producción y comercialización de la sal y de una incipiente agricultura y ganadería. En el año 1970 la parroquia Salinas inicia un crecimiento vertiginoso mediante la producción de materia prima, la diversificación de la producción y el emprendimiento social de sus habitantes y con él con el apoyo de clérigos, misioneros italianos y organizaciones no gubernamentales.

En el año 1992 se crea en la parroquia Salinas, el grupo de microempresas "El Salinerito", con el objetivo fortalecer a las organizaciones asociadas de emprendedores de la parroquia y darle un valor agregado a la materia prima, además de posicionar en el mercado nacional e internacional la marca "El Salinerito", que al inicio consistía en productos lácteos que eran producidos de manera artesanal a los que se les fueron agregando productos derivados del cacao, lana, confitería etc.,

La organización de economía comunitaria y solidaria se ha ido fortaleciendo a través de los años mediante un modelo de desarrollo productivo vasado en valores de trabajo, ahorro, cooperación, cooperativismo, diversificación de la producción, adecuada distribución de sus productos, reinversión de las utilidades en beneficio de la población y un alto grado de compromiso de sus pobladores, que han generado bienestar y buen vivir y convirtieron a la parroquia Salinas de Guaranda en un modelo económico exitoso.

\section{Métodos}

Los tipos de investigaciones que se utilizaron para llevar a cabo esta investigación son: la investigación exploratoria, la investigación descriptiva, e investigación documental requeridos para desarrollar el marco teórico del modelo de desarrollo de las microempresas del grupo "El Salinerito" en el Ecuador. Para (Cazau, 2006) "En la investigación exploratoria buscamos información sobre algún tema o problema por resultarnos relativamente desconocido", la investigación exploratoria nos proporciona las posibles variables a investigar, en cuanto a la investigación documental (Martínez, 2017) indica "La investigación documental es un tipo de estudio de interrogantes que emplea documentos oficiales y personales como fuente de información; dichos documentos pueden ser de diversos tipos: impresos, electrónicos o gráficos". Indica (Galán, 2011) "El objetivo de la investigación documental es elaborar un marco teórico conceptual para formar un cuerpo de ideas sobre el objeto de estudio y descubrir respuestas a determinados interrogantes a través de la aplicación de procedimientos documentales". "El Diseño de investigación descriptiva es un método científico que implica observar y describir el comportamiento de un sujeto sin influir sobre él de ninguna manera" (Shuttleworth, 2013).

\section{Resultados}

La parroquia Salinas del cantón Guaranda, provincia de Bolívar, está ubicada en la sierra central del Ecuador tiene una población de 7.262 habitantes, está en un rango altitudinal de 4.449 
metros sobre el nivel del mar y ocupa una superficie de 46.530 hectáreas, limita al Norte con la parroquia Simiatug y Facundo Vela, al Sur con la parroquia urbano Guanujo de Guaranda, al Este con la provincia de Tungurahua, y al Oeste con la parroquia San Luis de Pambil, Las Naves y Echeandía.. Antes de la colonia española fue conquistada por los incas y habitada por los Tomabelas de la etnia de los Chimbos, su nombre se debe a las minas de sal que se encuentra cercanas a la cabecera parroquial. Entre los siglos XVII y XVIII la corona española expropiar los terrenos pertenecientes a los indígenas y se los entrega a los conquistadores. En 1861 mediante una escritura de venta el general Juan José Flores y su esposa, la Señora Mercedes Jijón entregan los terrenos de hacienda Talahua, Mondonguera y Sinde al Señor Domingo Cordovez, propiedad, que en el transcurso de los años fue extendiéndose. En 1976, con la muerte de Alfredo Cordovez, empieza el proceso de litigios intrafamiliares, esto ocasiona la transferencia hacia nuevos propietarios.

En 1970 inicia un nuevo proceso de desarrollo económico comunitario que hasta esa fecha consistía en una incipiente explotación de las minas de sal. A esto se sumó la iniciativa de la misión salesiana y sus voluntarios que aprovecharon las iniciativas locales y la organización comunitaria y proponen un nuevo modelo de desarrollo para fortalecer la producción y la comercialización de la parroquia.

Los hitos o acontecimientos significativos que han marcado los momentos más importantes en el desarrollo de este proceso o modelo de desarrollo de las microempresas del grupo "El Salinerito" en el Ecuador están resumidos en la siguiente tabla:

Tabla 1. Hitos en el desarrollo de las microempresas del grupo "El Salinerito” en el Ecuador

\begin{tabular}{|c|c|c|}
\hline Año & Hito & Impacto positivo \\
\hline 1971 & $\begin{array}{l}\text { Inauguración casa comunal y sistema de agua en } \\
\text { la cabecera parroquial. }\end{array}$ & $\begin{array}{l}\text { Organización y sistema administrativo sinónimo de } \\
\text { progreso. }\end{array}$ \\
\hline 1972 & $\begin{array}{l}\text { Fundación de la Cooperativa de Ahorro y } \\
\text { Crédito Salinas Ltda. }\end{array}$ & $\begin{array}{l}\text { Apoyo para el desarrollo económico a través de } \\
\text { créditos. }\end{array}$ \\
\hline 1974 & $\begin{array}{l}\text { Construcción de carretera: Salinas la Palma y } \\
\text { Chazojuan, }\end{array}$ & $\begin{array}{l}\text { Soporte para la movilidad y transporte de productos y } \\
\text { personas. }\end{array}$ \\
\hline 1974 & Implementación de primera quesera. & Agroindustria para dinamizar la economía local. \\
\hline 1977 & Electrificación en la cabecera parroquial. & Mejora la calidad de vida de la población. \\
\hline 1980 & $\begin{array}{l}\text { Implementación de la planta de embutidos y } \\
\text { cárnicos de Salinas. }\end{array}$ & Desarrollo económico y creación fuentes de empleo. \\
\hline 1984 & $\begin{array}{l}\text { Inauguración del Colegio Técnico Agropecuario } \\
\text { Salinas }\end{array}$ & Acceso a la educación secundaria de la población. \\
\hline 1987 & $\begin{array}{l}\text { Apertura de la industria textil a cargo de } \\
\text { FUNORSAL }\end{array}$ & $\begin{array}{l}\text { Nueva alternativa industrial para generación de } \\
\text { ingresos y empleo. }\end{array}$ \\
\hline 1988 & $\begin{array}{l}\text { Reconocimiento de parroquia Eclesiástica de } \\
\text { Salinas }\end{array}$ & $\begin{array}{l}\text { La presencia del primer párroco, se proscribe las } \\
\text { costumbres y fiestas profanas. }\end{array}$ \\
\hline 1990 & Fundación de taller artesanal. & Genera empleo juvenil. \\
\hline
\end{tabular}




\begin{tabular}{|c|c|c|}
\hline 1992 & $\begin{array}{l}\text { Funcionamiento de la empresa de confites el } \\
\text { "Salinerito" }\end{array}$ & Nuevas plazas de trabajo. \\
\hline 1997 & Legalización de la Fundación Familia Salesiana. & Soporte positivo para el progreso de la parroquia \\
\hline 1998 & $\begin{array}{l}\text { Fundación del Colegio Técnico Particular } \\
\text { Bilingüe de Lanzaurco. }\end{array}$ & $\begin{array}{l}\text { Inserción de la población juvenil a la educación } \\
\text { secundaria de las comunidades. }\end{array}$ \\
\hline 2006 & $\begin{array}{l}\text { Nace la Corporación de Desarrollo Comunitario } \\
\text {-GRUPPO SALINAS-. }\end{array}$ & Fortalecimiento a micro empresas. \\
\hline 2007 & Servicio del internet inalámbrico. & $\begin{array}{l}\text { La población adquiere conocimientos de } \\
\text { comunicación y tecnología }\end{array}$ \\
\hline 2007 & Construcción de Mercado en el Centro parroquial & Viabiliza la comercialización de los productores. \\
\hline 2007 & $\begin{array}{l}\text { Doble tratamiento superficial bituminoso Cuatro } \\
\text { Esquinas }\end{array}$ & Vía asfaltada facilita movilidad \\
\hline 2012 & Mejoramiento de la Plaza Cívica & Espacios de fomento socio-cultural. \\
\hline 2013 & Unidad Educativa del Milenio & Nueva alternativa para la educación de calidad. \\
\hline 2013 & $\begin{array}{l}\text { Construcción de una oficina de MAGAP-B en la } \\
\text { cabecera parroquial. }\end{array}$ & $\begin{array}{l}\text { Servicios de asistencia técnica agropecuaria para los } \\
\text { productores. }\end{array}$ \\
\hline
\end{tabular}

Nota: Datos obtenidos del anuario 2016 "Salinerito”, economía solidaria.

\section{i. Modelo de desarrollo}

El modelo de desarrollo del grupo de microempresas "El Salinerito se basa en la economía social y solidaria que propone un modelo asociativo descentralizado basado en el ahorro y en una economía colectiva y solidaria que va más allá del beneficio o la utilidad empresarial sino apuesta a darle valor al trabajo y mejorar el nivel de vida de las personas mediante la reinversión de las utilidades de las empresas productivas en obras sociales que tiene como finalidad el desarrollo y la atención a las familias de la comunidad. El Art. 1 de la Ley Orgánica de la Economía Popular y Solidaria y Del Sector Financiero Popular y Solidario define a la economía popular y solidaria como: ". la forma de organización económica, donde sus integrantes, individual o colectivamente, organizan y desarrollan procesos de producción, intercambio, comercialización, financiamiento y consumo de bienes y servicios, para satisfacer necesidades y generar ingresos, basadas en relaciones de solidaridad, cooperación y reciprocidad, privilegiando al trabajo y al ser humano como sujeto y fin de su actividad, orientada al buen vivir, en armonía con la naturaleza, por sobre la apropiación, el lucro y la acumulación de capital”.

(Jácome, 2013) indica que "El desarrollo de Salinas está basado en los principios del cooperativismo y la economía solidaria. Son pilares fundamentales, contar con un grupo humano organizado para la producción comunitaria, otorgar créditos productivos a bajo costo, la no repartición de utilidades individuales, la colaboración colectiva y la rendición de cuentas puntual y permanente".

El modelo de desarrollo del grupo de microempresas el "Salinerito", se basa principalmente en la organización comunitaria de los pobladores de la parroquia Salinas, que 
impulsan proyectos de emprendimiento social mediante la concepción de la economía popular y solidaria.

\section{ii. Finalidad del grupo de microempresa el Salinerito}

\section{Tabla 2. Miembros del "Gruppo Salinas"}

\begin{tabular}{|c|c|}
\hline Institución & Finalidad \\
\hline $\begin{array}{c}\text { FUNORSAL (Fundación de } \\
\text { Organizaciones Campesinas } \\
\text { de Salinas) }\end{array}$ & $\begin{array}{l}\text { Es una entidad Jurídica sin fines de lucro creada mediante Acuerdo Ministerial No } \\
145 \text { del MAGAP con fecha } 27 \text { de Abril de } 1995 \text {, con el objeto de apoyar a las } \\
\text { organizaciones de base, crear, gestionar y desarrollar empresas y microempresas } \\
\text { para la producción, industrialización y comercialización de productos y servicios } \\
\text { procurando el desarrollo económico y social de los grupos y organizaciones } \\
\text { campesinas de Salinas de Guaranda. }\end{array}$ \\
\hline & $\begin{array}{l}\text { La Fundación es una organización social sin fines de lucro, legalmente constituida } \\
\text { y reconocida por el Ministerio de Inclusión Económica y Social mediante Acuerdo }\end{array}$ \\
\hline $\begin{array}{l}\text { FFSS (Fundación Familia } \\
\text { Salesiana Salinas) }\end{array}$ & $\begin{array}{l}\text { Ministerial } \mathrm{N}^{\mathrm{o}} 0486 \text { del } 1 \text { de febrero de } 2002 \text {. El objetivo de la Fundación se } \\
\text { encamina a la atención a niños, jóvenes, mujeres, adultos mayores y personas con } \\
\text { discapacidad, sobre todo a los más pobres a través de los servicios pastorales y } \\
\text { mediante la creación de actividades para generar empleo; al mejoramiento a la } \\
\text { calidad educativa en toda la parroquia. Cuenta con varias actividades para el } \\
\text { autofinanciamiento de su labor social como la fábrica de confites (Elaboración de } \\
\text { pasta de cacao, turrones de maní y macadamia, mermelada de mortiño, chocolates } \\
\text { en diferentes presentaciones), la fábrica de Aceites esenciales (Infusiones, pomadas, } \\
\text { aceites esenciales de diferentes plantas, shampoo, entre otros); varios talleres para } \\
\text { la capacitación y la inserción juvenil en el mundo laboral como el taller de alimentos } \\
\text { procesados (soya y sus derivados, mermeladas, confites, fideos, galletas, majar, } \\
\text { etc.), }\end{array}$ \\
\hline
\end{tabular}

FUGJS (Fundación Grupo Juvenil Salinas)

COACSAL (Cooperativa de Ahorro y Crédito "Salinas Ltda.")

TEXSAL (Asociación de Desarrollo Social de Artesanas Texsal Salinas)

PRODUCOOP

(Cooperativa de Producción Agropecuaria "El Salinerito")
Sostener la personería jurídica y la empresa de deshidratados en sociedad con la Fundación Familia Salesiana Salinas.

La Cooperativa de Ahorro y Crédito Salinas Ltda. Es una institución sólida, pionera en la prestación de servicios financieros, a lo largo de la parroquia y sus comunidades aledañas, que lidera una red de instituciones locales comprometidas con el desarrollo de su gente

Esta organización inicia en el año de 1974 como una actividad de la Cooperativa de Ahorro y Crédito, en marzo del año 2003 se legaliza como Asociación de Desarrollo Social de Artesanos Texal Salinas. En 1987 con la presencia de la hilandería en Salinas se facilitó la provisión de los hilos de variados colores y fibras naturales (oveja, llama y alpaca). Actualmente la Hilanderia Salinas continua siendo el único proveedor de materia prima.

La Cooperativa de Producción Agropecuaria "El Salinerito" está constituida con 183 socios legalmente registrados en la SEPS y 60 proveedores de materias primas. Su actividad principal es la elaboración de quesos. En la actualidad está constituida por 22 queseras. 


\begin{tabular}{cl}
\hline CONA (Comercializadora & $\begin{array}{l}\text { Dentro el GRUPPO SALINAS existen dos empresas comercializadoras de } \\
\text { productos "El Salinerito", una a nivel nacional denominada CONA } \\
\text { Nacional) y Centro de }\end{array}$ \\
$\begin{array}{c}\text { Exportaciones } \\
\text { (Comercializadora Nacional) y otra, el Centro de Exportaciones, encargada de la }\end{array}$ & promoción y ventas a nivel internacional.
\end{tabular}

Nota: Datos obtenidos del anuario 2016 "Salinerito", economía solidaria.

\section{iii. Analisis estadistico}

De acuerdo a los datos proporcionados por el Anuario 2016 Salinerito economia solidaria, con relacion a las ventas: en el 2011 fueron de USD \$ 904.923, en el 2012 de USD \$ 954.812, en el 2013 de USD \$ 1.035.209, en el 2014 de USD \$ 1.049.619, en el 2015 de USD \$ 1.222.152, y en el 2016 de USD \$ 1.147.899. Entre el 2011 y el 2012 hubo un incremento de las ventas del $5.51 \%$, entre el año 2012 y el 2013 hubo un incremento de 8.42\%, entre los años 2013 y 2014 existió un incremento 1.39\%, entre los años 2014 y 2015 el incremento fue del 16.44\%, mientras que entre los años 2015 y 2016 existió una disminución de las ventas del - 3.92\%. Los resultados muestran que las ventas desde 2011 han ido en aumento y por ende existe un crecimiento sostenido de la actividad productiva.

\section{Conclusiones}

El modelo de desarrollo del grupo de microempresas "El Salinerito" se fundamenta en el trabajo colectivo de los pobladores de la parroquia Salinas, con una planificación basada en valores como el trabajo, ahorro, autogestión, reinversión de sus utilidades y el emprendimiento social.

El emprendimiento social de la parroquia Salinas tiene como finalidad mejorar el nivel de vida de sus pobladores mediante la reinversión de las utilidades de las empresas productivas en obras sociales.

El progreso agroindustrial de la parroquia Salinas y el grupo de microempresas "El Salinerito", se debe a la organización comunitaria y solidaria comprometida de sus pobladores, a la planificación de la producción y la adecuada aplicación de los canales de distribución y comercialización de sus productos.

\section{Bibliografía}

Anuario 2016. "Salinerito" economía solidaria, obtenido de: http://www.salinerito.com/images/PUBLICACIONES/anuario2016.pdf

Cazau (2006), Introducción a la investigación en ciencias sociales, obtenido de: http://alcazaba.unex.es/asg/400758/Materiales/Introducci\%C3\%93n\%20a\%201a\%20inves tigaci\%C3\%93n\%20en\%20cc.Ss..pdf

Cobián C. (2013). ¿Qué es el emprendimiento social?, recuperado de: http://conprmetidos.org/que-es-el-empredimiento-social/ 
Corebusiness (2015). Emprendimiento en el Ecuador, recuperado de: http://www.ekosnegocios.com/revista/pdfTemas/1133.pdf

Dávila (2016), emprendimiento y gestión, obtenido de: https://educacion.gob.ec/wpcontent/uploads/downloads/2016/08/MINEDU_Libro_Emprendimiento-2-BGU-ilovepdfcompressed.pdf

Galán (2011). La investigación documental, obtenido de: http://manuelgalan.blogspot.com/2011/09/la-investgacion-documental_1557.html

Gallardo Y. Moreno A. (1999), Modulo 3: Recolección de la información, recuperado de http://www.unilibrebaq.edu.co/unilibrebaq/images/CEUL/mod3recoleccioninform.pdf

Hirschman, A. O. (1958). The strategy of economic development. New Have: Yale University Press.

Issuu.com (Agosto de 2013), Salinas Pueblo de Economía Solidaria, recuperado de: https://issuu.com/salinerito/docs/folleto_explicativo_salinas

Jácome (2013). El Salinerito un ejemplo de economía solidaria obtenido de: :http://www.revistalideres.ec/lideres/salinerito-ejemplo-economiasolidaria.html.

Jacome W. (2013). El Salinerito un ejemplo de economía solidaria, recuperado de:http://www.revistalideres.ec/lideres/salinerito-ejemplo-economia solidaria.html

Lasio V., Caicedo G., Ordeñana X., Izquierdo E. (2015). Global Entrepreneurship Monitor Ecuador 2015, recuperado de: http://www.espae.espol.edu.ec/images/documentos/publicaciones/libros/GemEcuador201 5.pdf

Martínez (2017). ¿Qué es la Investigación Documental? Características Principales, obtenido de: https://www.lifeder.com/investigacion-documental/

Shuttleworth, (2013), Diseño de Investigación Descriptiva, obtenido de: https://explorable.com/es/diseno-de-investigacion-descriptiva 